\title{
Use of Repetitive Transcranial Magnetic Stimulation for Treatment in Psychiatry
}

\author{
André Aleman \\ Department of Neuroscience, University Medical Center Groningen, University of Groningen, Groningen, the Netherlands
}

\begin{abstract}
The potential of noninvasive neurostimulation by repetitive transcranial magnetic stimulation (rTMS) for improving psychiatric disorders has been studied increasingly over the past two decades. This is especially the case for major depression and for auditory-verbal hallucinations in schizophrenia. The present review briefly describes the background of this novel treatment modality and summarizes evidence from clinical trials into the efficacy of rTMS for depression and hallucinations. Evidence for efficacy in depression is stronger than for hallucinations, although a number of studies have reported clinically relevant improvements for hallucinations too. Different stimulation parameters (frequency, duration, location of stimulation) are discussed. There is a paucity of research into other psychiatric disorders, but initial evidence suggests that rTMS may also hold promise for the treatment of negative symptoms in schizophrenia, obsessive compulsive disorder and post-traumatic stress disorder. It can be concluded that rTMS induces alterations in neural networks relevant for psychiatric disorders and that more research is needed to elucidate efficacy and underlying mechanisms of action.
\end{abstract}

KEY WORDS: Transcranial magnetic stimulation; Depression; Schizophrenia; Hallucinations; Prefrontal cortex.

\section{INTRODUCTION}

Despite numerous research efforts to develop novel treatments in psychiatry, the number of new effective treatments introduced over last decades is disappointing. One of the few novel treatment approaches that have received empirical support concerns transcranial magnetic stimulation (TMS). It was first proposed as a treatment for depression in the early 1990's and has been termed a "paradigm shift in psychiatry" as it uses noninvasive and nonconvulsive circuit-based physiological processes to treat psychiatric symptoms. ${ }^{1)}$ The approval of repetitive TMS (rTMS) as a treatment for depression by the United States Food and Drug Administration in 2008 has fueled interest from researchers and clinicians alike.

In this review, after briefly introducing the fundamentals of TMS, including a brief discussion of safety and suitability, I will summarize evidence from clinical trials in major depressive disorder (MD) and in auditory-verbal hallucinations in schizophrenia. These are the two only

Received: July 9, 2012 / Revised: April 15, 2013

Accepted: May 17, 2013

Address for correspondence: André Aleman, $\mathrm{PhD}$

Department of Neuroscience, University Medical Center Groningen,

University of Groningen, Antonius Deusinglaan 2, 9713AW

Groningen, the Netherlands

Tel: +31-503638798, Fax: +31-503638755

E-mail: a.aleman@med.umcg.nl psychiatric domains for which more than 10 randomized trials have been reported. ${ }^{2,3)}$ I will then discuss a number of issues that deserve attention when designing an rTMS trial or when evaluating published trials. These concern frequency of stimulation, location, duration and blinding.

Modern TMS was introduced in $1985^{4)}$ and has since then increasingly been applied for the study of brain regions involved in cognitive processing ${ }^{5)}$ and for treatment of psychiatric and neurological symptoms. The principle of brain stimulation with TMS is based on Faraday's law of induction for time-varying currents. More specifically, a time-varying magnetic field is generated by a current pulse through a stimulator coil placed over the scalp. A rapidly changing external magnetic field induces electric current intracranially. This way, the rapid rise and fall of the magnetic field induces a flow of current in the underlying brain tissue, and thus neural activation. ${ }^{6}$ The diameter of the induced field is of approximately $2-3 \mathrm{~cm}$, the same figure holds for the depth of stimulation, thus only cortical regions can be stimulated directly. Several neuroimaging studies have confirmed activation of underlying brain areas after TMS, for example over the motor cortex ${ }^{7}$ or over the prefrontal cortex. Speer et al. ${ }^{8)}$ observed increased blood flow (as measured with $\mathrm{H}_{2} \mathrm{O}$ positron emission tomography) after $10 \mathrm{~Hz}$ rTMS to the left dorsolateral prefrontal cortex (DLPFC), whereas $1 \mathrm{~Hz}$ stimulation de-

(c) This is an Open-Access article distributed under the terms of the Creative Commons Attribution Non-Commercial License (http://creativecommons.org/licenses/by-nc/3.0) which permits unrestricted non-commercial use, distribution, and reproduction in any medium, provided the original work is properly cited. 
creased blood flow. Indeed, frequencies of $1 \mathrm{~Hz}$ or lower are generally considered to be inhibitory (i.e., they reduce cortical excitability of the underlying area), whereas frequencies of $5 \mathrm{~Hz}$ and higher are considered to be excitatory. ${ }^{\text {) }}$

Although the exact mechanism of action of rTMS remains to be elucidated, there is evidence that dopaminergic neurotransmission is involved, at least for rTMS over prefrontal and motor areas. Such evidence comes from animal models ${ }^{9)}$ as well as human studies. ${ }^{10,11)}$ These studies have shown increased dopamine transmission in subcortical areas, but also in medial prefrontal areas, after TMS.

Intensity of stimulation is usually set at a certain percentage of the individual motor threshold. Motor threshold refers to the strength of the stimulus provided, which is the percentage of the total machine output that is required to produce movement of thumb or fingers. With regard to safety, intensities that are considerably higher than the motor threshold of a participant are associated with a higher risk of inducing an epileptic seizure (at least for rTMS at frequencies $>1 \mathrm{~Hz}$ ). It is important to note, however, that when precautions listed in internationally agreed guidelines ${ }^{12,13)}$ are taken into account, rTMS appears to be safe and well tolerated. Contraindications for TMS are pacemaker, aneurysm clip, heart/vascular clip, prosthetic valve, intracranial metal prosthesis, personal or familial history of epilepsy, medications that reduce the threshold for seizure, and high alcohol or drug consumption. Pregnant women and young children are also excluded from research studies, although they might be subject to TMS for clinical or therapeutic purposes. Since the introduction of the international safety guidelines occurrence of seizures has been very rare. Side effects are generally mild. They include transient headache (that responds well to analgesics), local discomfort as a consequence of direct stimulation of the facial musculature, and transient changes in the auditory threshold. The latter can be avoided by using earplugs, which is recommended.

\section{MAIN SUBJECTS}

\section{rTMS for Depression}

The rationale for applying rTMS over the prefrontal cortex for the treatment of depression was derived from the hypothesis of an imbalanced relationship between prefrontal cortical regions and limbic regions (including ventral cingulate, insula, amygdala and hippocampus) underlying dysregulated mood. ${ }^{14)}$ More specifically, evidence of hypometabolism of the left DLPFC was hypothesized to underlie reduced cognitive control of emotion, which has been confirmed by neuroimaging studies. ${ }^{15-17)}$ The dorsolateral frontal areas are thought to play a pivotal role in affect regulation, for example by cognitive reappraisal of negative stimuli. A study that applied rTMS to the left DLPFC in healthy subjects ${ }^{18)}$ found less positive affect and more monotonous speech, as is characteristic for depression. This suggests that stimulation of the left DLPFC does not merely improve positive mood indistinctively, but concerns a key node of the emotion regulation network. Interfering with coordinated processing in this area through rTMS in normal, healthy functioning brains could does cause imbalances in stead of restoring function. In depressed patients, however, the circuit may be dysfunctional due to a decreased activation of the DLPFC.

A typical rTMS treatment of depression includes a 20to 40 -minute session delivering 3,000 to 6,000 pulses at 10 $\mathrm{Hz}, 5$ days a week for 4 to 8 weeks. ${ }^{1)}$ Integration of the published evidence supports the efficacy of rTMS in depression. In a comprehensive meta-analysis of randomized, sham-controlled studies of rTMS treatment for depression, Slotema et $a l^{2}{ }^{2}$ recently calculated a mean weighted effect size of $0.55(p<0.001)$, which is in the moderate range of magnitude. This was based on 34 published studies, encompassing a total of 1,562 patients. Previous meta-analyses had also documented a positive and statistically significant effect size of rTMS compared to sham stimulation. ${ }^{19,20)}$ Berlim et al. ${ }^{21)}$ recently reported meta-analytical integration of response, remission and drop-out rates following high-frequency rTMS for MD. Data from 29 randomized controlled trials were included, totaling 1,371 patients. They reported that, after approximately 13 sessions, $29.3 \%$ and $18.6 \%$ of subjects receiving rTMS were classified as responders and remitters, respectively. This was threefold of those receiving sham rTMS: $10.4 \%$ and $5 \%$, respectively. The pooled odds ratio (OR) was $3.3(p<0.0001)$ for both response and remission rates. Associated number needed to treat (NNT) were 6 and 8 , respectively. These results certainly warrant their conclusion that rTMS is associated with clinically relevant antidepressant effects. Moreover, the studies in the meta-analysis reported a benign tolerability profile. Interestingly, a meta-analysis ${ }^{22)}$ of studies using low-frequency rTMS $(1 \mathrm{~Hz}$, in general) to the frontal cortex in MD patients, also observed an improvement: $34.6 \%$ and $9.7 \%$ of subjects receiving active rTMS and sham rTMS were classified as remitters $(\mathrm{OR}=4.76, p<0.0001$; 
$\mathrm{NNT}=5$ ). However, this concerned only 8 trials with 263 participants. Thus, the evidence base is limited and in need of thorough replication. The findings do pose the question, however, whether high-frequency rTMS is excitatory (i.e., activating the frontal cortex), and what the mechanism is behind similar effects on depression for low-frequency rTMS.

Most studies of rTMS in depression included a treatment-resistant group, in which it is notably difficult to obtain clinical improvement. In an interesting study, George et $a .^{23)}$ reported a multi-center rTMS trial of 190 antidepressant drug-free patients with unipolar nonpsychotic MD. They received $10 \mathrm{~Hz}$ rTMS to the left prefrontal cortex at $120 \%$ motor threshold for 37.5 minutes $(3,000$ pulses per session) using a figure-eight solid-core coil with 3 weeks of daily weekday treatment. Trains of rTMS were of a 4-second train duration, and 26-second intertrain interval. Primary efficacy analysis revealed a significant effect of treatment on the proportion of remitters $(14.1 \%$ active rTMS and 5.1\% sham). The odds of attaining remission were 4.2 times greater with active rTMS than with sham. NNT was 12 . Thus, rTMS is not only relevant for treatment-resistant groups but can also be of use in improving depression in other groups of patients.

\section{rTMS for Hallucinations}

The first study into rTMS for auditory-verbal hallucinations in schizophrenia was published by Hoffman et $a l .{ }^{24-26)}$ They targeted the left temporoparietal cortex, which converges with Wernicke's area, the speech perception area. This area has been shown to be hyperactive in neuroimaging studies of hallucinations. ${ }^{27-29)}$ The idea is to reduce this increased excitability of left temporoparietal cortex by stimulating daily with $1 \mathrm{~Hz}$ over this area. In their study with 50 patients, half was randomly assigned to rTMS for 9 days (with a total of 132 min of stimulation), and the other half to sham TMS (by rotating the coil with 45 degrees). In the rTMS condition, 52\% improved in severity and frequency of hallucinations, whereas in the control group this was only $17 \%$. The effect of rTMS lasted for 13 weeks, on average.

In a meta-analysis published in 2007, we integrated effect sizes of published studies using a sham-controlled design and rTMS over the left temporoparietal area. ${ }^{30)}$ Most of these studies used comparable treatment parameters: same location, intensity between $80-100 \%$ of motor threshold and frequency of $1 \mathrm{~Hz}$. Duration of treatment varied between studies but was between 4 and 10 days, with daily sessions of 15-20 min of rTMS. Only one center included two daily sessions of rTMS. ${ }^{31,32)}$ The mean effect size was $d=0.76$, which represents a large effect. The effect did not generalize to other positive symptoms, and was specific for hallucinations. Slotema et al. ${ }^{2}$ included 7 studies (they disregarded cross-over designs and only included parallel group designs) in their meta-analysis of rTMS effects on hallucinations and found a smaller effect size, $d=0.54$, a medium effect size that was statistically significant.

However, not all studies included in these meta-analyses found an improvement of hallucinations after rTMS. For example, McIntosh et $a l^{33)}$ and Fitzgerald et al. ${ }^{34)}$ failed to find significant reduction of hallucination severity or frequency in the rTMS versus sham condition comparison. This could be partly due to low power (e.g., the effect size of Fitzgerald et al. $^{34)}$ was 0.47 in the direction of improvement with rTMS), but certainly also because a substantial part of the patients do not respond. Indeed, three recent studies also showed very modest effects of rTMS or even a lack of improvement. ${ }^{35-37)}$ Of note, Blumberger et al. ${ }^{37)}$ did not stimulate the left temporoparietal cortex (Wernicke's area) but left auditory cortex (Heschl's gyrus). One of the largest studies to date ${ }^{36)}$ failed to find an improvement of rTMS on hallucinations. However, the study still included less than 25 patients per group, implying modest power. That is, the differences in effect sizes reported by the authors may have been statistically significant with larger samples. A recent meta-analy$\mathrm{sis}^{38)}$ integrated these three new studies with the previously published trials and reported a smaller effect size than earlier meta-analyses, albeit still statistically significant, $d=0.44$. The effect was no longer significant at one month of follow-up.

A number of studies also investigated stimulation of the right hemisphere $(\mathrm{RH})$, as neuroimaging studies have also implied aberrant activation of right temporal cortex during auditory-verbal hallucinations. ${ }^{28,39)}$ However, studies that included RH stimulation did not obtain stronger reductions of hallucination severity than has been reported for left hemisphere. ${ }^{35,40)}$ In the study by Vercammen et al., ${ }^{35)}$ the condition with RH stimulation also involved left hemisphere stimulation, and was thus bilateral, i.e. patients received $10 \mathrm{~min}$ of left hemisphere stimulation followed by 10 min of RH stimulation. Although this design makes sense because neuroimaging studies have shown increased activation on both sides, ${ }^{28,39)}$ such a stimulation could also be problematic due to the inhibitory connections between homotopic areas. ${ }^{41)}$ That is, inhibition through $1 \mathrm{~Hz}$ rTMS of the RH area may indirectly lead to 
excitation due inhibition of callosal inhibition. ${ }^{42)}$

\section{Frequency and Intensity of Stimulation}

With regard to the frequency of stimulation, most studies for depression used $10 \mathrm{~Hz}$ and most for hallucinations used $1 \mathrm{~Hz}$. This is consistent with the idea of $10 \mathrm{~Hz}$ being excitatory and $1 \mathrm{~Hz}$ being inhibitory. Notably, one study applied $10 \mathrm{~Hz}$ to the left hemisphere DLPFC and $1 \mathrm{~Hz}$ to the right DLPFC for treatment of depression. ${ }^{43)}$ The rationale for this was that $1 \mathrm{~Hz}$ to the $\mathrm{RH}$ may inhibit the $\mathrm{RH}$ but, through inhibitory callosal connections, lead to activation of the left hemisphere. The results were consistent with this line of reasoning, in the sense that this bilateral condition showed considerable improvement of depression.

However, the difference between slow $(1 \mathrm{~Hz})$ and fast $(>5 \mathrm{~Hz})$ rTMS may not be so clear-cut. For example, Montagne-Larmurier et al. ${ }^{44)}$ used $20 \mathrm{~Hz}$ rTMS for improving hallucinations in schizophrenia and reported beneficial effects.

With regard to intensity of stimulation, most studies stimulate at $100 \%$ of motor threshold or lower, to ensure safety (a slightly higher risk of seizures may be present at higher thresholds). However, in a study of 185 patients, Johnson et $a l .{ }^{45)}$ concluded that stimulation at $120 \%$ of motor threshold appears safe for a broad range of patients. Intensities above $100 \%$ of motor threshold may be more effective for treatment. ${ }^{1)}$

\section{Location of stimulation}

The most used method to localize the DLPFC area for antidepressant rTMS without the help of neuronavigation is the "5-cm method". In this procedure, the motor cortical site is localized that stimulates the abductor pollicis brevis muscle of the contralateral hand, and then one moves $5 \mathrm{~cm}$ anteriorly along the scalp surface in order to target the DLPFC. It has been shown, however, that this method may yield a location that is too posterior (i.e., targeting premotor cortex) in part of the patients. ${ }^{46)}$ Indeed, a number of studies have reported that using neuronavigation to determine the key area within the DLPFC, yields superior antidepressant efficacy compared to the 5-cm method. ${ }^{46-48)}$

Neuronavigated rTMS has also been applied for coil positioning in the treatment of hallucinations. More specifically, a number of studies ${ }^{49-50)}$ applied functional magnetic resonance imaging (MRI) scans to localize activated areas during hallucinations indivually in schizophrenia patients. They then navigated to those areas for coil placement. Hoffman et al. ${ }^{49)}$ reported that delivering rTMS to left temporoparietal sites was more effective in reducing hallucination severity than stimulation of other locations. Sommer et al. ${ }^{50)}$ compared the functional MRI (fMRI)guided rTMS to the conventional positioning using the 10-20 electrode system (area in between T3 and P3). They observed equal improvement in both conditions. Finally, Slotema et al. ${ }^{38)}$ did not find a statistical superiority of fMRI-guided rTMS over and above the conventional method or sham stimulation. It should be noted that a limitation of the fMRI-guided method is that it relies on functional scans made during hallucination periods in the scanner (patients press a button to indicate periods of hallucination). However, most patients do not hallucinate with neatly alternating periods of hallucination and nonhallucination activity, which is required for statistical analysis of these scans. Thus, it is very difficult to apply this in a majority of patients. A more convenient method may be to localize speech perception cortex using a language task (e.g., Schönfeldt-Lecuona et al. ${ }^{51)}$ ).

\section{Duration of Treatment and Effects}

Studies differ widely in terms of duration of treatment, either defined as the number of sessions in which rTMS was delivered or the number of days or weeks the treatment was continued. For example, with regard to hallucination treatment, Hoffman et al. ${ }^{25)}$ stimulated each patient for 9 days with a total of 7,920 pulses, whereas Vercammen et al. ${ }^{35)}$ stimulated for 6 days, but with longer sessions and with two sessions a day, totalling 14,400 pulses per patient. Thus, although the former trial had a longer duration in terms of treatment days, the number of pulses was almost half of those in the latter trial. However, whereas for hallucinations no systematic relationship has been observed between duration of treatment and efficacy, this is certainly the case for depression. That is, studies that applied rTMS for 3 weeks or more have generally observed stronger improvements than shorter treatments. ${ }^{52)}$ It should be noted that the evidence base for hallucination treatment studies is small, whereas for depression much more studies with larger numbers of participants have been conducted. Thus, a firm conclusion can not be reached for the hallucination treatment, but for depression it seems safe to conclude that treatments should last preferably 4 weeks or more. ${ }^{1)}$

Another important question regards the duration of treatment effects. Unfortunately, most studies only report immediate post-treatment effects, without including a follow-up after periods of one month, 6 months or longer. For depression, two large trials found that, after 6 months, on- 
ly $12-14 \%$ of patients had relapsed (cited in the article of George and Post ${ }^{1)}$ ). For hallucinations, Hoffman et al. ${ }^{25)}$ reported a mean duration of survivorship of 13.1 weeks (defined as return of hallucinations to $80 \%$ of baseline severity). Thus, rTMS for depression seems to have a longer-lasting effect than for hallucinations in schizophrenia. It goes without saying that more research is needed with longitudinal designs to evaluate the duration of treatment response and clinical factors that can help predict such duration.

\section{rTMS in Other Psychiatric Disorders}

Other conditions for which rTMS has been studied as a potential novel therapy include negative symptoms in schizophrenia, obsessive compulsive disorder (OCD), post-traumatic stress disorder (PTSD). For negative symptoms in schizophrenia, a meta-analysis ${ }^{53)}$ of nine trials, involving 213 patients, revealed a significant improvement that was stronger for rTMS than for sham. The overall mean weighted effect size was in the small-to-medium range, $d=0.43$. Studies with a longer duration of treatment ( $\geq 3$ weeks) had a larger mean effect size when compared to studies with a shorter treatment duration. These studies typically stimulated the left DLPFC, as in studies of depression, but one could argue that the right DLPFC is also of relevance as it has been implicated in negative symptoms. Future studies may thus also include the right DLPFC as a target of neurostimulation.

Only a few studies have been directed at investigating rTMS for OCD. Jaafari et al. ${ }^{54)}$ reviewed 12 studies published up to 2010, including open and randomized, sham-controlled trials. They concluded that two brain regions showed potential for improvement of OCD symptoms after rTMS: the supplementary motor area and the orbitofrontal cortex. In a study that was published after this review, Gomes et al. ${ }^{55)}$ assigned 22 patients with OCD to either rTMS or sham over the supplementary motor area bilaterally, for two weeks, with a 3-month follow-up. At follow-up, patients receiving active rTMS showed, on average, a $35 \%$ reduction on the Y-BOCS, as compared with a $6.2 \%$ reduction in those receiving sham treatment.

Even less studies have investigated rTMS for PTSD. Watts et al. ${ }^{56)}$ randomly assigned 20 patients with PTSD to receive either $10 \mathrm{rTMS}$ sessions delivered at $1 \mathrm{~Hz}$ to the right DLPFC or 10 sham rTMS sessions to the same area. The rTMS group improved significantly more than the sham group on two measures of PTSD symptoms, with a symptom reduction of approximately $30 \%$. Clearly, larger studies are needed to confirm such effects.

\section{Blinding}

It is notoriously difficult to perform rTMS treatment in a double blind design. The reason for this is that the person delivering the TMS can easily become aware of the treatment condition. This is by definition the case when the sham condition involves rotation of the coil. But also when placebo-coils are used a distinction between real and placebo TMS can readily be made on the basis of muscle twitches in the face, scalp area or extremities (in case of motor cortex stimulation for determination of motor threshold). The patient can also notice differences: until recently placebo coils did not give any scalp sensation during stimulation, whereas the opposite is true for real TMS. This problem has been addressed in recent years however. For example, George et al. ${ }^{23)}$ in their depression trial applied sham rTMS with a coil with a metal insert blocking the magnetic field and scalp electrodes that delivered matched somatosensory sensations. Thus, the patients experienced scalp sensations that were similar to those produced by the real TMS stimulation.

The issue of blinding is of particular relevance as robust placebo effects have been shown in rTMS trials of depression $^{57)}$ and blinding has been shown to be partly unsuccessful. ${ }^{58)}$ That is, people in the real rTMS groups were significantly more likely to think they had received real rTMS compared with those in sham rTMS groups.

\section{CONCLUSION}

TMS is a novel treatment strategy in psychiatry for which evidence is accumulating that it can help a subset of patients. Specifically, $10 \mathrm{~Hz}$ rTMS over the left DLPFC can improve depression. The evidence base for rTMS to reduce auditory-verbal hallucinations is less strong, due to a smaller number of studies. However, meta-analyses suggest a statistically significant improvement of rTMS compared to sham. More research is needed to corroborate this, preferably in multi-center designs to ensure larger samples. Research should also focus on negative symptoms of schizophrenia, for which some promising results have been reported and on other conditions such as OCD and PTSD. Finally, the neural basis of rTMS deserves further investigation using pre- and posttreatment fMRI and positron emission tomography scanning to elucidate the mechanism of action.

\section{REFERENCES}

1. George MS, Post RM. Daily left prefrontal repetitive transcranial magnetic stimulation for acute treatment of medication-resistant depression. Am J Psychiatry 2011;168:356- 
364

2. Slotema CW, Blom JD, Hoek HW, Sommer IE. Should we expand the toolbox of psychiatric treatment methods to include Repetitive Transcranial Magnetic Stimulation (rTMS)? A meta-analysis of the efficacy of rTMS in psychiatric disorders. J Clin Psychiatry 2010;71:873-884.

3. Matheson SL, Green MJ, Loo C, Carr VJ. Quality assessment and comparison of evidence for electroconvulsive therapy and repetitive transcranial magnetic stimulation for schizophrenia: a systematic meta-review. Schizophr Res 2010;118:201-210.

4. Barker AT, Jalinous R, Freeston IL. Non-invasive magnetic stimulation of human motor cortex. Lancet 1985;325:11061107.

5. Sack AT. Transcranial magnetic stimulation, causal structure-function mapping and networks of functional relevance. Curr Opin Neurobiol 2006;16:593-599.

6. Hallett M. Transcranial magnetic stimulation and the human brain. Nature 2000;406:147-150.

7. Siebner HR, Takano B, Peinemann A, Schwaiger M, Conrad B, Drzezga A. Continuous transcranial magnetic stimulation during positron emission tomography: a suitable tool for imaging regional excitability of the human cortex. Neuroimage 2001;14:883-890.

8. Speer AM, Kimbrell TA, Wassermann EM, D Repella J, Willis MW, Herscovitch $\mathrm{P}$, et al. Opposite effects of high and low frequency rTMS on regional brain activity in depressed patients. Biol Psychiatry 2000;48:1133-1141.

9. Keck ME, Welt T, Müller MB, Erhardt A, Ohl F, Toschi $\mathrm{N}$, et al. Repetitive transcranial magnetic stimulation increases the release of dopamine in the mesolimbic and mesostriatal system. Neuropharmacology 2002;43:101-109.

10. Strafella AP, Paus T, Barrett J, Dagher A. Repetitive transcranial magnetic stimulation of the human prefrontal cortex induces dopamine release in the caudate nucleus. $J$ Neurosci 2001;21:RC157.

11. Cho SS, Strafella AP. rTMS of the left dorsolateral prefrontal cortex modulates dopamine release in the ipsilateral anterior cingulate cortex and orbitofrontal cortex. PLoS One 2009;4:e6725

12. Wassermann EM. Risk and safety of repetitive transcranial magnetic stimulation: report and suggested guidelines from the International Workshop on the Safety of Repetitive Transcranial Magnetic Stimulation, June 5-7, 1996. Electroencephalogr Clin Neurophysiol 1998;108:1-16.

13. Rossi S, Hallett M, Rossini PM, Pascual-Leone A; Safety of TMS Consensus Group. Safety, ethical considerations, and application guidelines for the use of transcranial magnetic stimulation in clinical practice and research. Clin Neurophysiol 2009;120:2008-2039.

14. Phillips ML, Drevets WC, Rauch SL, Lane R. Neurobiology of emotion perception II: Implications for major psychiatric disorders. Biol Psychiatry 2003;54:515-528.

15. Sackeim HA, Prohovnik I, Moeller JR, Brown RP, Apter S, Prudic J, et al. Regional cerebral blood flow in mood disorders. I. Comparison of major depressives and normal controls at rest. Arch Gen Psychiatry 1990;47:60-70.

16. Martinot JL, Hardy P, Feline A, Huret JD, Mazoyer B, Attar-Levy D, et al. Left prefrontal glucose hypometabolism in the depressed state: a confirmation. Am J Psychiatry 1990;147:1313-1317.

17. Elliott R, Baker SC, Rogers RD, O'Leary DA, Paykel ES, Frith $\mathrm{CD}$, et al. Prefrontal dysfunction in depressed patients performing a complex planning task: a study using positron emission tomography. Psychol Med 1997;27:931-942.
18. Barrett J, Della-Maggiore V, Chouinard PA, Paus T. Mechanisms of action underlying the effect of repetitive transcranial magnetic stimulation on mood: behavioral and brain imaging studies. Neuropsychopharmacology 2004;29:11721189.

19. Schutter DJ. Antidepressant efficacy of high-frequency transcranial magnetic stimulation over the left dorsolateral prefrontal cortex in double-blind sham-controlled designs: a meta-analysis. Psychol Med 2009;39:65-75.

20. Dell'osso B, Camuri G, Castellano F, Vecchi V, Benedetti $\mathrm{M}$, Bortolussi S, et al. Meta-review of metanalytic studies with repetitive transcranial magnetic stimulation (rTMS) for the treatment of major depression. Clin Pract Epidemiol Ment Health 2011;7:167-177.

21. Berlim MT, van den Eynde F, Tovar-Perdomo S, Daskalakis $\mathrm{ZJ}$. Response, remission and drop-out rates following high-frequency repetitive transcranial magnetic stimulation (rTMS) for treating major depression: a systematic review and meta-analysis of randomized, double-blind and shamcontrolled trials. Psychol Med 2013. [Epub ahead of print]

22. Berlim MT, Van den Eynde F, Jeff Daskalakis Z. Clinically meaningful efficacy and acceptability of low-frequency repetitive transcranial magnetic stimulation (rTMS) for treating primary major depression: a meta-analysis of randomized, double-blind and sham-controlled trials. Neuropsychopharmacology 2013:38:543-551.

23. George MS, Lisanby SH, Avery D, McDonald WM, Durkalski V, Pavlicova M, et al. Daily left prefrontal transcranial magnetic stimulation therapy for major depressive disorder: a sham-controlled randomized trial. Arch Gen Psychiatry 2010;67:507-516.

24. Hoffman RE, Boutros NN, Hu S, Berman RM, Krystal JH, Charney DS. Transcranial magnetic stimulation and auditory hallucinations in schizophrenia. Lancet 2000;355:10731075.

25. Hoffman RE, Hawkins KA, Gueorguieva R, Boutros NN, Rachid F, Carroll K, et al. Transcranial magnetic stimulation of left temporoparietal cortex and medication-resistant auditory hallucinations. Arch Gen Psychiatry 2003;60:4956.

26. Hoffman RE, Gueorguieva R, Hawkins KA, Varanko M, Boutros NN, Wu YT, et al. Temporoparietal transcranial magnetic stimulation for auditory hallucinations: safety, efficacy and moderators in a fifty patient sample. Biol Psychiatry 2005;58:97-104.

27. Silbersweig DA, Stern E, Frith C, Cahill C, Holmes A, Grootoonk S, et al. A functional neuroanatomy of hallucinations in schizophrenia. Nature 1995;378:176-179.

28. Shergill SS, Brammer MJ, Williams SC, Murray RM, McGuire PK. Mapping auditory hallucinations in schizophrenia using functional magnetic resonance imaging. Arch Gen Psychiatry 2000;57:1033-1038.

29. Allen P, Larøi F, McGuire PK, Aleman A. The hallucinating brain: a review of structural and functional neuroimaging studies of hallucinations. Neurosci Biobehav Rev 2008;32: 175-191.

30. Aleman A, Sommer IE, Kahn RS. Efficacy of slow repetitive transcranial magnetic stimulation in the treatment of resistant auditory hallucinations in schizophrenia: a metaanalysis. J Clin Psychiatry 2007;68:416-421.

31. Poulet E, Brunelin J, Bediou B, Bation R, Forgeard L, Dalery J, et al. Slow transcranial magnetic stimulation can rapidly reduce resistant auditory hallucinations in schizophrenia. Biol Psychiatry 2005;57:188-191.

32. Brunelin J, Poulet E, Bediou B, Kallel L, Dalery J, D'amato 
$\mathrm{T}$, et al. Low frequency repetitive transcranial magnetic stimulation improves source monitoring deficit in hallucinating patients with schizophrenia. Schizophr Res 2006;81: 41-45.

33. McIntosh AM, Semple D, Tasker K, Harrison LK, Owens DG, Johnstone EC, et al. Transcranial magnetic stimulation for auditory hallucinations in schizophrenia. Psychiatry Res 2004;127:9-17.

34. Fitzgerald PB, Benitez J, Daskalakis JZ, Brown TL, Marston NA, de Castella A, et al. A double-blind sham-controlled trial of repetitive transcranial magnetic stimulation in the treatment of refractory auditory hallucinations. J Clin Psychopharmacol 2005;25:358-362.

35. Vercammen A, Knegtering H, Bruggeman R, Westenbroek $\mathrm{HM}$, Jenner JA, Slooff CJ, et al. Effects of bilateral repetitive transcranial magnetic stimulation on treatment resistant auditory-verbal hallucinations in schizophrenia: a randomized controlled trial. Schizophr Res 2009;114:172179.

36. Slotema CW, Blom JD, de Weijer AD, Diederen KM, Goekoop R, Looijestijn J, et al. Can low-frequency repetitive transcranial magnetic stimulation really relieve medicationresistant auditory verbal hallucinations? Negative results from a large randomized controlled trial. Biol Psychiatry 2011;69:450-456.

37. Blumberger DM, Christensen BK, Zipursky RB, Moller B, Chen R, Fitzgerald PB, et al. MRI-targeted repetitive transcranial magnetic stimulation of Heschl's gyrus for refractory auditory hallucinations. Brain Stimul 2012;5:577-585.

38. Slotema CW, Aleman A, Daskalakis ZJ, Sommer IE. Meta-analysis of repetitive transcranial magnetic stimulation in the treatment of auditory verbal hallucinations: update and effects after one month. Schizophr Res 2012;142:40-45.

39. Lennox BR, Park SB, Medley I, Morris PG, Jones PB. The functional anatomy of auditory hallucinations in schizophrenia. Psychiatry Res 2000;100:13-20.

40. Jandl M, Steyer J, Weber M, Linden DE, Rothmeier J, Maurer $\mathrm{K}$, et al. Treating auditory hallucinations by transcranial magnetic stimulation: a randomized controlled cross-over trial. Neuropsychobiology 2006;53:63-69.

41. Lee SH, Kim W, Chung YC, Jung KH, Bahk WM, Jun TY, et al. A double blind study showing that two weeks of daily repetitive TMS over the left or right temporoparietal cortex reduces symptoms in patients with schizophrenia who are having treatment-refractory auditory hallucinations. Neurosci Lett 2005;376:177-181.

42. Cook ND. The brain code; mechanisms of information transfer and the role of the corpus callosum. London:Methuen; 1986.

43. Fitzgerald PB, Benitez J, de Castella A, Daskalakis ZJ, Brown TL, Kulkarni J. A randomized, controlled trial of sequential bilateral repetitive transcranial magnetic stimulation for treatment-resistant depression. Am J Psychiatry 2006; 163:88-94.

44. Montagne-Larmurier A, Etard O, Razafimandimby A, Morello $\mathrm{R}$, Dollfus S. Two-day treatment of auditory hallucinations by high frequency rTMS guided by cerebral imaging: a 6 month follow-up pilot study. Schizophr Res 2009;113:77-83.

45. Johnson KA, Baig M, Ramsey D, Lisanby SH, Avery D, McDonald WM, et al. Prefrontal rTMS for treating de- pression: location and intensity results from the OPT-TMS multi-site clinical trial. Brain Stimul 2013;6:108-117.

46. Rusjan PM, Barr MS, Farzan F, Arenovich T, Maller JJ, Fitzgerald PB, et al. Optimal transcranial magnetic stimulation coil placement for targeting the dorsolateral prefrontal cortex using novel magnetic resonance image-guided neuronavigation. Hum Brain Mapp 2010;31:1643-1652.

47. Schönfeldt-Lecuona C, Lefaucheur JP, Cardenas-Morales L, Wolf RC, Kammer T, Herwig U. The value of neuronavigated rTMS for the treatment of depression. Neurophysiol Clin 2010;40:37-43.

48. Trojak B, Meille V, Chauvet-Gelinier JC, Bonin B. Further evidence of the usefulness of MRI-based neuronavigation for the treatment of depression by rTMS. $J$ Neuropsychiatry Clin Neurosci 2011;23:E30-E31.

49. Hoffman RE, Hampson M, Wu K, Anderson AW, Gore JC, Buchanan RJ, et al. Probing the pathophysiology of auditory/verbal hallucinations by combining functional magnetic resonance imaging and transcranial magnetic stimulation. Cereb Cortex 2007;17:2733-2743.

50. Sommer IE, de Weijer AD, Daalman K, Neggers SF, Somers $\mathrm{M}, \mathrm{Kahn}$ RS, et al. Can fMRI-guidance improve the efficacy of rTMS treatment for auditory verbal hallucinations? Schizophr Res 2007;93:406-408.

51. Schönfeldt-Lecuona C, Grön G, Walter H, Büchler N, Wunderlich A, Spitzer M, et al. Stereotaxic rTMS for the treatment of auditory hallucinations in schizophrenia. Neuroreport 2004;15:1669-1673.

52. Gross M, Nakamura L, Pascual-Leone A, Fregni F. Has repetitive transcranial magnetic stimulation (rTMS) treatment for depression improved? A systematic review and meta-analysis comparing the recent vs. the earlier rTMS studies. Acta Psychiatr Scand 2007;116:165-173.

53. Dlabac-de Lange JJ, Knegtering R, Aleman A. Repetitive transcranial magnetic stimulation for negative symptoms of schizophrenia: review and meta-analysis. J Clin Psychiatry 2010;71:411-418.

54. Jaafari N, Rachid F, Rotge JY, Polosan M, El-Hage W, Belin $\mathrm{D}$, et al. Safety and efficacy of repetitive transcranial magnetic stimulation in the treatment of obsessive-compulsive disorder: a review. World J Biol Psychiatry 2012;13: 164-177.

55. Gomes PV, Brasil-Neto JP, Allam N, Rodrigues de Souza E. A randomized, double-blind trial of repetitive transcranial magnetic stimulation in obsessive-compulsive disorder with three-month follow-up. J Neuropsychiatry Clin Neurosci 2012;24:437-443.

56. Watts BV, Landon B, Groft A, Young-Xu Y. A sham controlled study of repetitive transcranial magnetic stimulation for posttraumatic stress disorder. Brain Stimul 2012;5:3843.

57. Brunoni AR, Lopes M, Kaptchuk TJ, Fregni F. Placebo response of non-pharmacological and pharmacological trials in major depression: a systematic review and meta-analysis. PLoS One 2009;4:e4824.

58. Broadbent HJ, van den Eynde F, Guillaume S, Hanif EL, Stahl D, David AS, et al. Blinding success of rTMS applied to the dorsolateral prefrontal cortex in randomised shamcontrolled trials: a systematic review. World J Biol Psychiatry 2011;12:240-248. 